\title{
Transient Plume Model Testing Using LADEE Spacecraft Attitude Control System Operations
}

\author{
M. S. Woronowicz
}

Stinger Ghaffarian Technologies, Inc., 7701 Greenbelt Rd., Greenbelt, Maryland 20770, USA.

\begin{abstract}
The Lunar Atmosphere Dust Environment Explorer (LADEE) spacecraft is being designed for a mission featuring low altitude orbits of the Moon to take relevant ambient measurements before that environment becomes altered by future exploration activities. Instruments include a neutral mass spectrometer capable of measuring ambient species density levels below 100 molecules $/ \mathrm{cm}^{3}$. Coincidentally, with a favorable combination of spacecraft orientations, it is also possible to measure plume gases from LADEE attitude control system thruster operations as they are reflected from the daytime lunar surface and subsequently intercepted by the spacecraft as it orbits overhead. Under such circumstances, it may be possible to test a variety of properties and assumptions associated with various transient plume models or to infer certain aspects regarding lunar surface properties.
\end{abstract}

Keywords: Plume Modeling, Lunar Exploration, Free Molecule Flow.

PACS: 51.10.+y

\section{INTRODUCTION}

Investigators analyzing mass transport issues associated with contamination control engineering for various spacecraft programs sometimes encounter modeled phenomena whose behaviors are very difficult to substantiate in detail through ground-based testing and measurement. One such phenomenon is associated with transient descriptions of plume expansions from chemical rockets into high vacuum. [1-3] Very few thermal vacuum facilities exist that are capable of keeping high-temperature combustion or decomposition gaseous products, such as hydrogen, from building up during thruster testing to the point that they subsequently disturb a free expansion or application-specific physical features of interest. [4]

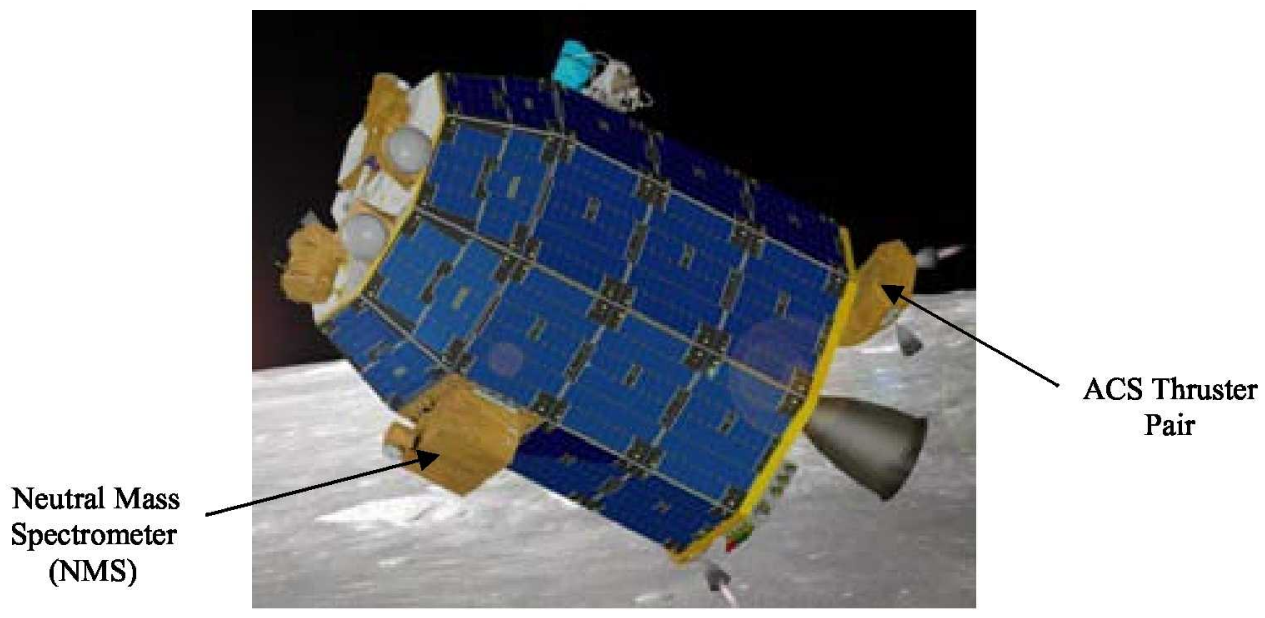

FIGURE 1. Lunar Atmosphere Dust Environment Explorer, featuring NMS instrument. 
The Lunar Atmosphere Dust Environment Explorer (LADEE) spacecraft is being designed for a mission featuring low altitude orbits of the Moon to take relevant ambient measurements before that environment becomes altered by future activities associated with human exploration (Fig. 1). Depending on orbital insertion timing, the observatory's mission could last over four months, beginning in 2013. [5,6] In some cases, the orbital altitude may dip as low as $20 \mathrm{~km}$ above the lunar surface. [5] LADEE's instrument suite includes a sensitive Neutral Mass Spectrometer (NMS) which will be oriented in the spacecraft's ram direction to measure tenuous atmospheric constituents. Ambient density levels vary widely based on local solar illumination, but may exceed $10^{5}$ molecules $/ \mathrm{cm}^{3}$ during a lunar dawn. [7] NMS will be able to detect individual species concentrations at much lower levels, however. [8]

LADEE's attitude control system (ACS) includes $5-1 b_{\mathrm{f}}$ bipropellant thrusters using monomethylhydrazine (MMH) fuel with MON-3 oxidizer, mounted in two canted pairs beneath the spacecraft's lower deck. [5] If the ACS units are directed forward and toward the lunar surface, it is possible for individual species associated with their operations, reflected from the daytime lunar surface, to be quantitatively observed by the NMS as the observatory subsequently passes overhead. At lower altitudes, thruster plume fluxes to the lunar surface are maximized, as are reflected fluxes back to the spacecraft; also NMS may intercept peak species fluxes before disturbances from lunar gravity exert much influence. In this manner, NMS measurements could be used to test a variety of characteristics associated with transient plume models, $[1,9,10]$ including evolution of species concentration (magnitudes, time-offlight measurements, angular distribution, species separation effects), thruster exit conditions, or to infer local elements of lunar surface properties such as permeation or gas-surface interaction models.

\section{TEST CASE CONDITIONS}

LADEE will orbit the Moon in a variety of orientations and orbit altitudes. With surface values of $1737 \mathrm{~km}$ for lunar radius $R \supset$ and a gravitational acceleration constant of $g D=1.62 \mathrm{~m} / \mathrm{s}^{2}$, the orbital velocity at $20 \mathrm{~km}$ circular altitude should be $V_{\text {orb }}=1.67 \mathrm{~km} / \mathrm{s}$. [7] For this test case, a featureless strip of lunar surface was modeled along the ground track having width $=120 \mathrm{~km}$, length $=270 \mathrm{~km}$.

For a brief thruster firing $20^{\circ}$ below the satellite's velocity vector with a bulk velocity of $V_{\mathrm{e}} \approx 3.0 \mathrm{~km} / \mathrm{s}$, one would expect the average period for this pulse of gas to reach the lunar surface to be about $19.2 \mathrm{~s}$ in a ballistic trajectory due to lunar gravity, and $19.5 \mathrm{~s}$ in a radial trajectory without this influence. For diffusely-reflected gas of a dominant bipropellant thruster combustion product such as water vapor from the lunar surface at a daylight temperature of $380 \mathrm{~K}$, [7] the time to reach $20 \mathrm{~km}$ based on its average thermal velocity and average direction would be $31.1 \mathrm{~s}$ with lunar gravity and $29.9 \mathrm{~s}$ without.

Since ballistic perturbations from radial trajectories increase with travel time, it was decided acceptable to approximate conditions by neglecting the influence of gravity and limiting the period of study to one minute. Orbit altitude and ACS thruster firing angle were chosen to produce easily measurable peaks of dominant ACS thruster gases within this period.

For the sake of simplicity, changes in LADEE's altitude due to ACS operations were neglected. NMS will generally face the ram direction when collecting measurements, so only molecules reaching LADEE's position from forward locations constitute the local ensemble density measured by NMS. Interactions between reflected plume gases and the ambient atmosphere (exosphere) were neglected since the level of rarefaction would be so considerable.

LADEE's attitude control system (ACS) includes four 5- $\mathrm{lb}_{\mathrm{f}}$ bipropellant thrusters using monomethylhydrazine (MMH) fuel and MON-3 oxidizer. Table 1 presents mass fractions for dominant species assumed herein. [11]

TABLE 1. Dominant ACS thruster plume species mass fractions. [11]

\begin{tabular}{|c|c|c|c|c|c|}
\hline Species & $\mathrm{N}_{2}$ & $\mathrm{H}_{2} \mathrm{O}$ & $\mathrm{CO}$ & $\mathrm{CO}_{2}$ & $\mathrm{H}_{2}$ \\
\hline Mass Fraction & 0.426 & 0.294 & 0.178 & 0.086 & 0.016 \\
\hline
\end{tabular}

For assumed performance parameters and frozen flow properties, exit conditions featured a bulk velocity $V_{\mathrm{e}} \approx 3.0$ $\mathrm{km} / \mathrm{s}$ and a static temperature $T_{\mathrm{e}} \approx 550 \mathrm{~K}$. In the test case studied, it was assumed two forward-facing ACS units would fire for one second at angles $20^{\circ}$ below LADEE's velocity vector towards the lunar surface. 


\section{MODEL FORMULATION}

In order to determine the magnitude of impact lunar surface-reflected ACS plume gases could have on NMS measurements, considering the vast scales of distances and levels of rarefaction involved, it was decided to use a set of point source, free molecule (FM) equations to approximately describe both the plume gas approaching the lunar surface as well as that diffusely reflected from it. [10] To develop this model, the collisionless Boltzmann equation had been solved for a directionally-constrained point source $Q_{1}$ meant to describe directed flow from a nozzle exit over $2 \pi$ steradians centered on the source normal.

$$
\begin{aligned}
& \frac{\partial f}{\partial t}+v \cdot \frac{\partial f}{\partial x}+g \cdot \frac{\partial f}{\partial v} \approx \frac{\partial f}{\partial t}+v \cdot \frac{\partial f}{\partial x}=Q_{1} ; \quad Q_{1}=\frac{2 \beta^{4}}{A_{1} \pi} \delta(x) \dot{m}(t)(v \cdot \hat{n}) \exp \left(-\beta^{2}\left(v-u_{\mathrm{e}}\right)^{2}\right) \\
& A_{1} \equiv e^{-s^{2} \cos ^{2} \phi_{\mathrm{e}}}+\sqrt{\pi} s \cos \phi_{\mathrm{e}}\left(1+\operatorname{erf}\left(s \cos \phi_{\mathrm{e}}\right)\right)
\end{aligned}
$$
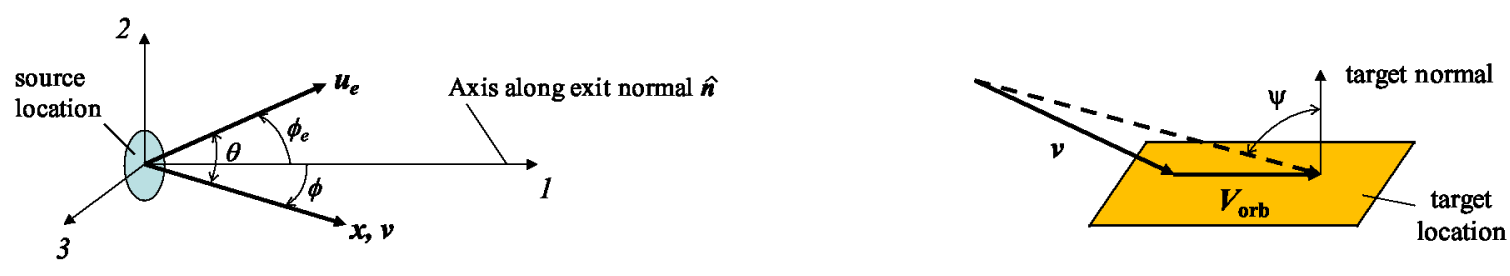

FIGURE 2. Schematic representation of various quantities and angles used in analytical model.

$Q_{1}$ represents directed flow from a Lambertian, thermal velocity distribution superimposed on convective exit velocity $u_{\mathrm{e}}$ for mass flow rate $\dot{m}, \beta \equiv 1 / \sqrt{2 R T}$, and speed ratio $s \equiv \beta u_{\mathrm{e}}$. Normal $\hat{\boldsymbol{n}}$ represents the orientation of a local starting surface element, and $\boldsymbol{v} \cdot \hat{\boldsymbol{n}}$ emphasizes the imposed directional constraint. Generally $\boldsymbol{u}_{\mathrm{e}}$ is not aligned with $\hat{n}$, the angle between the two being defined by $\phi_{\mathrm{e}}$ (Fig. 2). Local angle $\phi$ is measured between variable position $\boldsymbol{x}$ (distance $r$, experiencing local velocity $\boldsymbol{v}$ ) and $\hat{\boldsymbol{n}}$, and angle $\theta$ is measured between $\boldsymbol{u}_{\mathrm{e}}$ and $\boldsymbol{x}$.

Another FM source had been developed by Narasimha in conjunction with an analytical study of flow through a circular orifice into high vacuum. [9] This source, denoted by $Q_{\mathrm{N}}$, is derived from assuming the free expansion of a suddenly unbounded gas:

$$
Q_{\mathrm{N}}=\frac{\beta^{3}}{\pi \sqrt{\pi}} \delta(x) \dot{m}(t) \exp \left(-\beta^{2}\left(v-u_{\mathrm{e}}\right)^{2}\right)
$$

Clearly $Q_{1}$ differs from $Q_{\mathrm{N}}$ by a directional constraint that produces a distinctly different character in the limit of $s=0$; expressions developed using $Q_{1}$ are consistent with outgassing or surface desorption, while those using $Q_{\mathrm{N}}$ lack directional dependence. Although a recent study demonstrated the consequences of substituting $Q_{\mathrm{N}}$ for $Q_{1}$ in analyzing molecular beam and pulsed laser ablation data, [10] it appeared possible to test the relative fidelity of these models using the NMS measurement scenario described above.

Earlier experience indicated that a single-second thruster pulse evolution could be replaced by an instantaneous pulse source. [1,2] When $\dot{m}$ in Eqns. (1) \& (2) is described by a Dirac Delta function $\Delta m \delta(t)$, one may solve for successive moments of $f$ to find pulse-mode response expressions for quantities like density $\rho$ and mass flux $\dot{\Phi}$. For fixed geometries with a target surface element at location $\boldsymbol{x}$ relative to the plume source, incident fluxes make angle $\psi$ with the local receiver normal, as shown in Fig. 2 above.

$$
\rho_{1}(x, t)=\frac{2 \Delta m \alpha^{4} \cos \phi}{A_{1} \pi r^{3}} e^{w^{2}-s^{2}} e^{-z^{2}} ; \quad \rho_{\mathrm{N}}(x, t)=\frac{\Delta m \alpha^{3}}{\pi \sqrt{\pi} r^{3}} e^{w^{2}-s^{2}} e^{-z^{2}}
$$




$$
\dot{\Phi}(\boldsymbol{x}, t)=\frac{\rho r}{t} \cos \psi
$$

In Eqns. (3a) and (3b), $z \equiv \alpha-w, \alpha \equiv \beta r / t$, and $w \equiv s \cos \theta$. For Eq. (4), one may substitute either form for density to obtain the corresponding mass flux. An impermeable, inert lunar surface was assumed for this test case, therefore species mass flux $\dot{\Phi}$, reflected from a local surface element with area $\Delta A$ at any given time $t$, was equal to that reaching it from the incident plume. The surface element center then became a new source using that incident flux in either Eq. (3a) or (3b) with magnitude $\Delta m \approx \dot{\Phi}(t) \Delta A \Delta t$ to create a contribution to the reflected density at all later timesteps measured at NMS moving with $V_{\text {orb }}$.

If desired, it would be possible to model the effect of a permeable lunar regolith and/or the effects of surface chemistry by replacing the mass conservation statement with suitable mathematical models. Other gas-surface interaction models could be substituted for Eqns. (3a) and (3b) to produce scattered reflected fluxes. In particular, Eq. (3a) is consistent with Maxwell's model for diffuse scattering. [3]

\section{RESULTS}

The test case was run using both $Q_{1}$ and $Q_{\mathrm{N}}$ type models in pulse mode. Results include logarithmic contour maps of overall mass density across a featureless section of the lunar surface from ACS operations, as well as transient plots of surface-reflected number density levels intercepted by a forward-facing NMS instrument. The contour maps take advantage of symmetry about the axis of LADEE's ground track.

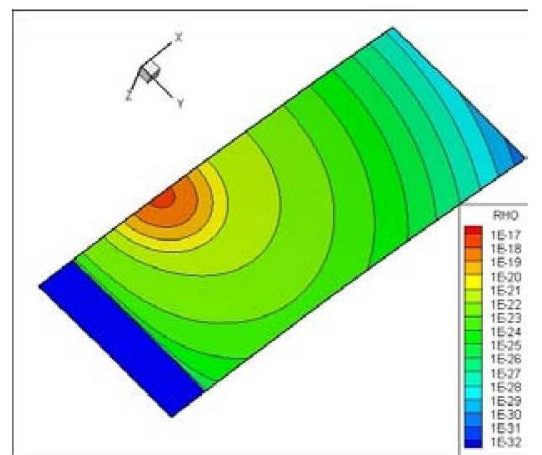

(a)

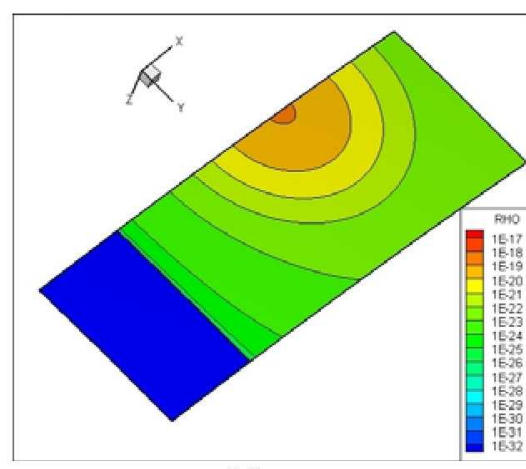

(b)

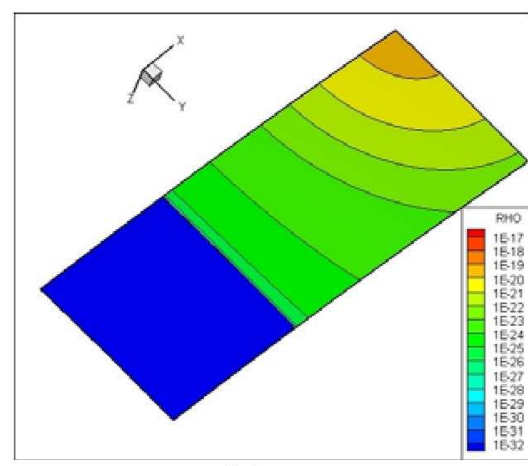

(c)

FIGURE 3. Test case mass density impingement contour maps at lunar surface after $Q_{1}$ pulse: (a) $20 \mathrm{~s}$, (b) $40 \mathrm{~s}, \&$ (c) $60 \mathrm{~s}$.

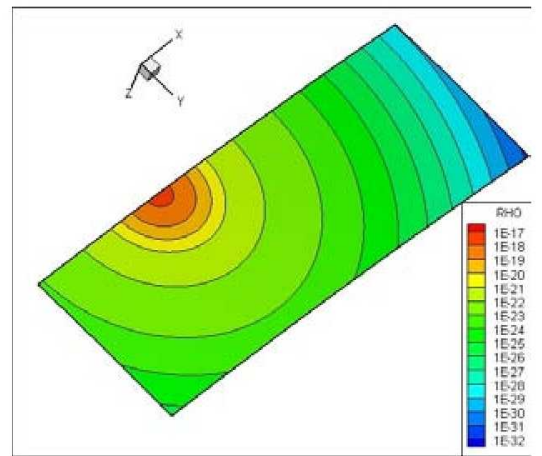

(a)

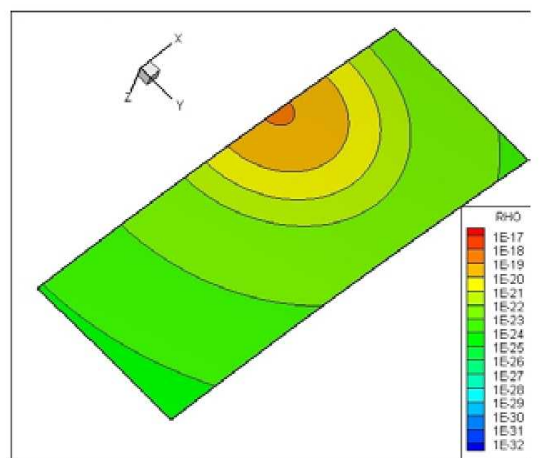

(b)

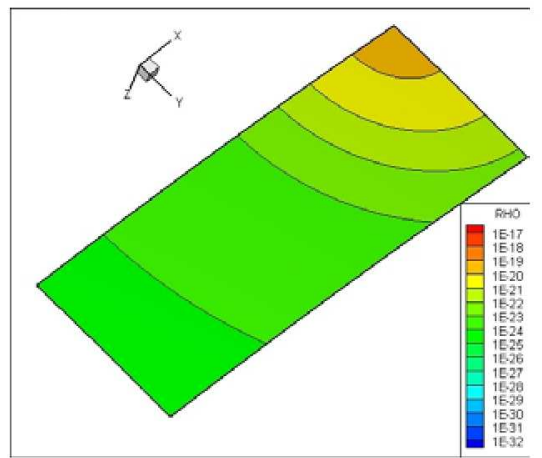

(c)

FIGURE 4. Test case mass density impingement contour maps at lunar surface after $Q_{\mathrm{N}}$ pulse: (a) $20 \mathrm{~s}$, (b) $40 \mathrm{~s}, \&$ (c) $60 \mathrm{~s}$.

In Fig. 3, a sequence of snapshots conveys the transient development of density across a featureless lunar surface due to a one-second LADEE ACS thruster pulse in 20 -second increments. A maximum peak value of $2.7 \times 10^{-15}$ 
$\mathrm{g} / \mathrm{cm}^{3}$ was reached along the centerline at $15 \mathrm{~s}$. Figure 3 also illustrates how the plume convected along LADEE's orbital direction as its footprint spread with time.

Each thruster was modeled using a single $Q_{1}$ source. Since these only provide non-zero values over $2 \pi$ steradians centered on the thruster nozzle axis, there is an abrupt cutoff in the contours that propagates at $V_{\text {orb }}$ due to this simplification. A more realistic analysis would incorporate locally varying conditions across a freezing surface capturing the thruster near-field expansion into the backflow regime. That data would be used to create a network of point sources to describe the expansion and create a better representation of the surface density map in that region.

Solutions for species mass fluxes were also computed to propagate their subsequent presence at the LADEE NMS aperture. They exhibit behavior similar to the evolution of density and are not presented here.

Figure 4 presents results using the $Q_{\mathrm{N}}$ source, which share surprising similarities to $Q_{1}$ results depicted in Fig. 3. Near the maximum flux location at the time of peak intensity for the $Q_{\mathrm{N}}$ source, the maximum density level was 96 percent as high as that for $Q_{1}$ results. However, it does not exhibit the cutoff for positions beyond right angles off the thruster plume axis. Since these are molecular flow models, one should wonder how a neutral gas flow directed forward via a nozzle can find itself at such high angles in the absence of any physical mechanism.

It was noticed that the elapsed times for peak individual species mass fluxes to reach the lunar surface using either $Q_{1}$ or $Q_{\mathrm{N}}$ type sources occurred more rapidly than expected (19-20 s) based on the geometry and nozzle exit speed, and it appears the basis for this is derived from the models themselves. Reviewing Eqns. (3) \& (4), noting that flux $\mathrm{M}$ varies as $t^{-\mathrm{D}}$, setting the time derivative to zero for peak flux yields

$$
t_{\max \text { flux }}=\frac{2 \beta r}{w\left(1+\sqrt{1+\frac{2 D}{w^{2}}}\right)} .
$$

For $w=s$ on the plume centerline, $t_{\max \text { flux }} \rightarrow r / V_{\mathrm{e}}$ as $s \rightarrow \infty$. For any finite value of $s$ however, the time to reach maximum flux actually decreases due to the component of kinetic energy associated with the non-zero thermal distribution. To observe this effect, it was decided to run a case using the $Q_{1}$ source where the nozzle exit static temperature $T_{\mathrm{e}}$ was decreased from $550 \mathrm{~K}$ to $55 \mathrm{~K}$. Results are depicted in Fig. 5 below. Differences between those results (Fig. 5) and Fig. 3 are much more substantial than those between Figs. $3 \& 4$. For the lower $T_{\mathrm{e}}$ case, peak fluxes tend to occur between 19-20 s, and flux distributions are more tightly concentrated about those peaks.

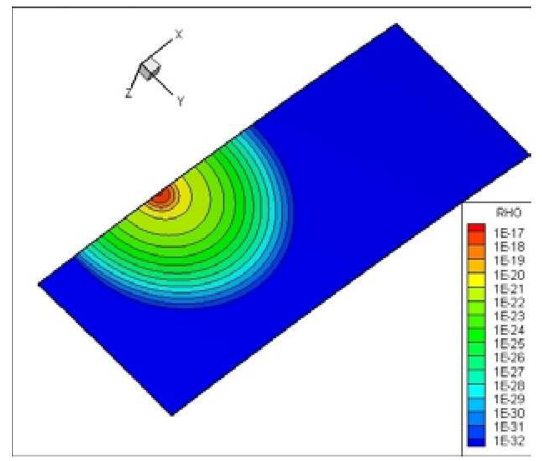

(a)

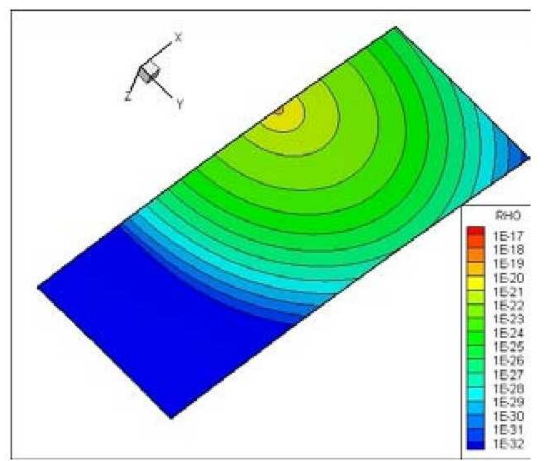

(b)

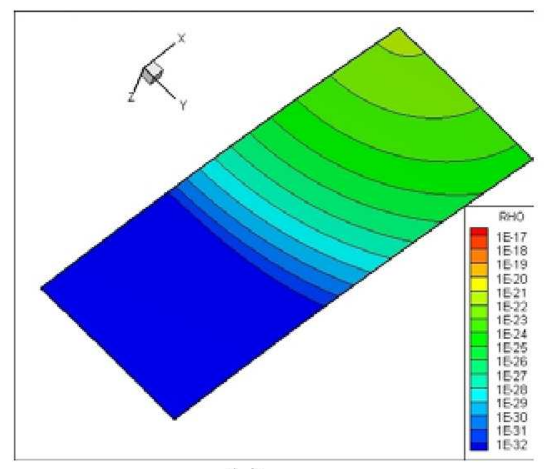

(c)

FIGURE 5. Surface mass density for $Q_{1}$ pulse, same conditions as Fig. 3, except for low $T_{\mathrm{e}}$ : (a) $20 \mathrm{~s}$, (b) $40 \mathrm{~s}, \&$ (c) $60 \mathrm{~s}$.

For the final phase of each run, lunar surface fluxes for each species at every timestep and location were used to create transient, thermally-accommodated, diffuse $(s=0), Q_{1}$ and $Q_{\mathrm{N}}$ sources to calculate their contributions to number density measured by the LADEE NMS as it would subsequently pass overhead. Results are presented in Fig. $6 \mathrm{a}\left(Q_{1}\right.$ vs. $\left.Q_{\mathrm{N}}\right)$ and Fig. $6 \mathrm{~b}\left(Q_{1}, T_{\mathrm{e}}=550 \mathrm{~K}\right.$ vs. $\left.T_{\mathrm{e}}=55 \mathrm{~K}\right)$ below.

In Fig. 6a, $Q_{1}$-generated estimates gave rise to higher levels than $Q_{\mathrm{N}}$ values, with greater discrepancies occurring for higher molecular weight species, along with steeper gradients. The basic deficit of $Q_{\mathrm{N}}$ fluxes relative to $Q_{1}$ is due to the nature of the $Q_{\mathrm{N}}$ source, which is spherically-symmetric for $s=0$. As a consequence, half its material is directed below the lunar surface, violating the conservation assumption for mass fluxes used in this study. Some investigators using $Q_{\mathrm{N}}$-type sources constrain the distribution function to exist only in the outbound hemisphere, [12] but this fix does not produce a Lambertian cosine distribution. Nevertheless, it could be difficult to distinguish which source model exhibits closer fidelity using NMS density measurements once such data becomes available. 
In Fig. $6 \mathrm{~b}$, the effect of higher speed ratio and fixed $V_{\mathrm{e}}$ is readily apparent. The delay in peak surface fluxes translates to a longer period before reaching maximum density levels, and the tighter footprint for lunar surface mass flux produces higher peak density levels once they do occur.

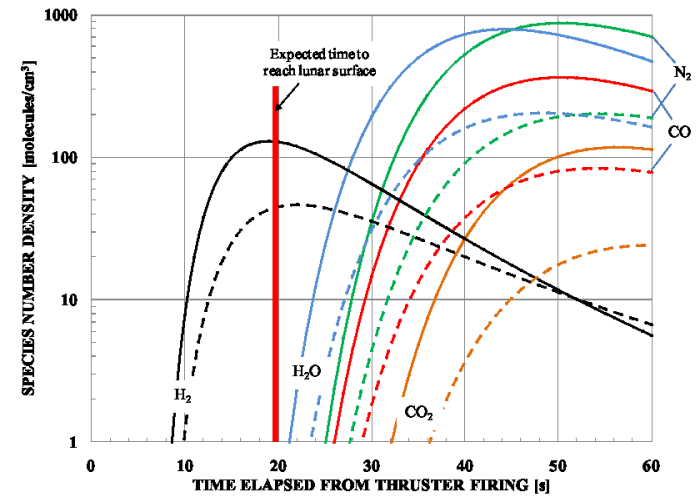

(a)

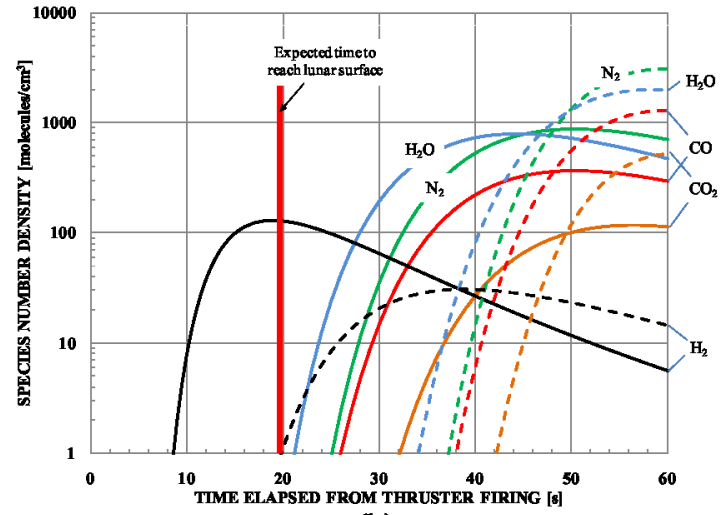

(b)

FIGURE 6. Comparison of predicted species contributions measured by LADEE NMS. (a) $Q_{1}$ (solid) vs. $Q_{\mathrm{N}}$ (dashed), (b) effect of $T_{\mathrm{e}}$ on $Q_{1}$; baseline $\left(T_{\mathrm{e}}=550 \mathrm{~K}\right.$, solid $)$ vs. $10 \times$ lower value $\left(T_{\mathrm{e}}=55 \mathrm{~K}\right.$, dashed).

\section{CONCLUDING REMARKS}

A study was performed to test the possibility that LADEE ACS thruster operations could be observed by its NMS instrument after reflection from the lunar surface. Results indicate such measurements are possible, and the study explored the possibility that the fidelity of various transient source flow plume models could be rated. It may also be possible to similar assessments for models describing the effects of a permeable lunar regolith, surface chemistry, and gas-surface interaction models.

\section{ACKNOWLEDGMENTS}

The author gratefully acknowledges support from NASA Contract NNG07CA21C, particularly the encouragement of Ms Sharon Straka, NASA-GSFC Code 546, and SGT, Inc.

\section{REFERENCES}

1. M. Woronowicz, Rarefied Gas Dynamics: $25^{\text {th }}$ International Symposium, Publishing House of the Siberian Branch of the Russian Academy of Sciences, Novosibirsk, 2007, pp. 853-858.

2. M. Woronowicz, $3^{\text {rd }}$ International Symposium on Formation Flying, Missions and Technologies, ESA Pub. SP654, 2008.

4. G. Dettleff, K. Plähn, Rarefied Gas Dynamics: $21^{\text {st }}$ International Symposium, Cepadues Editions, Toulouse, 1999,8 pp.

5. M. Mecham, Aviation Week \& Space Technology, 172, No. 3, 18 January 2010, pp. 25-26.

6. Lunar Atmosphere and Dust Environment Explorer, NASA National Space Science Data Center, <http://nssdc.gsfc.nasa.gov/ nmc/spacecraftDisplay.do?id=LADEE>, Dr. Ed Grayzeck, 23 November 2009, last retrieved 25 January 2010.

7. G. Heiken, et al., Lunar Sourcebook-A User's Guide to the Moon, Cambridge University Press, NY, 1991, pp. $28,40-5$.

8. LADEE Payload System Design Review, NASA-Goddard Space Flight Center, 17 June 2009.

9. R. Narasimha, Journal of Fluid Mechanics, 12, 294-308 (1962).

10. M. Woronowicz, Rarefied Gas Dynamics: $26^{\text {th }}$ International Symposium, AIP, 1084, Melville, NY, 2009 , pp. $565-70$.

11. H. Trinks, R. Hoffman, AIAA Paper No. 83-1447, Montreal, QC, Jun. 1983.

12. O. Rovenskaya, I. Voronich, Rarefied Gas Dynamics: $25^{\text {th }}$ International Symposium, Publishing House of the Siberian Branch of the Russian Academy of Sciences, Novosibirsk, 2007, pp. 304-309. 